\title{
Hemorrhagic Brain Metastases: A Diagnostic Dilemma and Diagnosis of Exclusion
}

\author{
Mukta Meel $^{1} \quad$ Mukesh Kumar Bhaskar $^{2} \quad$ Arpita Jindal ${ }^{1}$ \\ ${ }^{1}$ Department of Pathology, SMS Medical College, Jaipur, Rajasthan, \\ India \\ ${ }^{2}$ Department of Neurosurgery, SMS Medical College and Attached \\ Hospital, Jaipur, Rajasthan, India \\ Indian J Neurosurg 2021;00:1-3
}

Address for correspondence Mukesh Kumar Bhaskar, MCh Department of Neurosurgery, SMS Medical College and Attached Hospital, Jaipur, Rajasthan, India (e-mail: drmannu84@gmail.com).

\author{
Abstract \\ Keywords \\ - hemorrhagic brain \\ metastasis \\ - lung carcinoma \\ - pleomorphic \\ carcinoma
}

Pleomorphic carcinoma is a malignant and aggressive primary lung carcinoma that occurs at a rate of approximately $0.3 \%$. This rarely encountered tumor may present a diagnostic challenge to neuroradiologists and pathologists, as it has propensity to present as hemorrhagic brain metastasis with unknown primary and result in delay in diagnosis that could impact clinical outcome. Herein, we report a unique case in its presentation in a 56-year-old female, having symptomatic brain metastasis prior to the discovery of the pulmonary lesion.

\section{Introduction}

Spontaneous intracerebral hemorrhage (ICH) is commonly caused by hypertension, advanced age, prior ICH, renal failure, use of anticoagulant drugs, cerebral amyloid angiopathy, and tumor. Tumor-related spontaneous ICH is relatively uncommon. The leading causes of tumor-related $\mathrm{ICH}$ are metastases of extracranial origin (36\%), followed by glioblastoma multiforme (30\%). ${ }^{1}$ In adults, the most common source of brain metastases is lungs. Pulmonary pleomorphic carcinoma (PC) is an infrequent tumor with an incidence rate ranging from $0.1 \%$ to $0.3 \%$ of all lung tumors and has a poor prognosis because surgery, irradiation, and chemotherapy are ineffective. ${ }^{2}$ Here, we report a rare case of PC lung in a 56-year-old female presenting as unknown primary as well as manifesting ICH, causing sudden unconsciousness with left-sided hemiparesis as well as multiple thrombi in anterior and middle cerebral artery. Thus, the current case highlights the importance of brain biopsy together with immunohistochemistry (IHC) for a case of unknown primary as well as rare tumor presentation.

\section{Case Report}

A 56-year-old female admitted in neurosurgery department with complaints of vomiting and sudden unconsciousness with left-sided hemiparesis. Patient was managed with supportive treatments. During hospitalization, preliminary blood investigations were done which were normal (normal coagulation function, total leukocyte count [TLC] and platelet count). On examination, patient had Glasgow coma scale (GCS) score E1V1M4. CT scan of brain showed a heterogeneous hypodense lesion of $4.5 \times 5 \times 4.8 \mathrm{~cm}$ in right parietal lobe with edema in right parieto-temporal-occipital lobe, and thalamoganglionic region causing effacement of right lateral ventricle, midline shift of $8 \mathrm{~mm}$ toward left side and right uncal herniation (-Fig. 1A). CT angiography of brain showed thrombi in anterior and middle cerebral artery. Urgent craniotomy was performed, and while evacuating hematoma, suspicious clotted tissue was seen and sent for pathological examination. Grossly, we received several gray-brown to gray-white soft-tissue pieces, collectively measuring $3 \times 2 \times 1 \mathrm{~cm}$. On histopathological examination,
DOI https://doi.org/ $10.1055 / \mathrm{s}-0041-1730873$ ISSN 2277-954X (c) 2022. Neurological Surgeons' Society of India.

This is an open access article published by Thieme under the terms of the Creative Commons Attribution-NonDerivative-NonCommercial-License, permitting copying and reproduction so long as the original work is given appropriate credit. Contents may not be used for commercial purposes, or adapted, remixed, transformed or built upon. (https://creativecommons.org/licenses/by-nc-nd/4.0/). Thieme Medical and Scientific Publishers Pvt. Ltd. A-12, 2nd Floor, Sector 2, Noida-201301 UP, India 


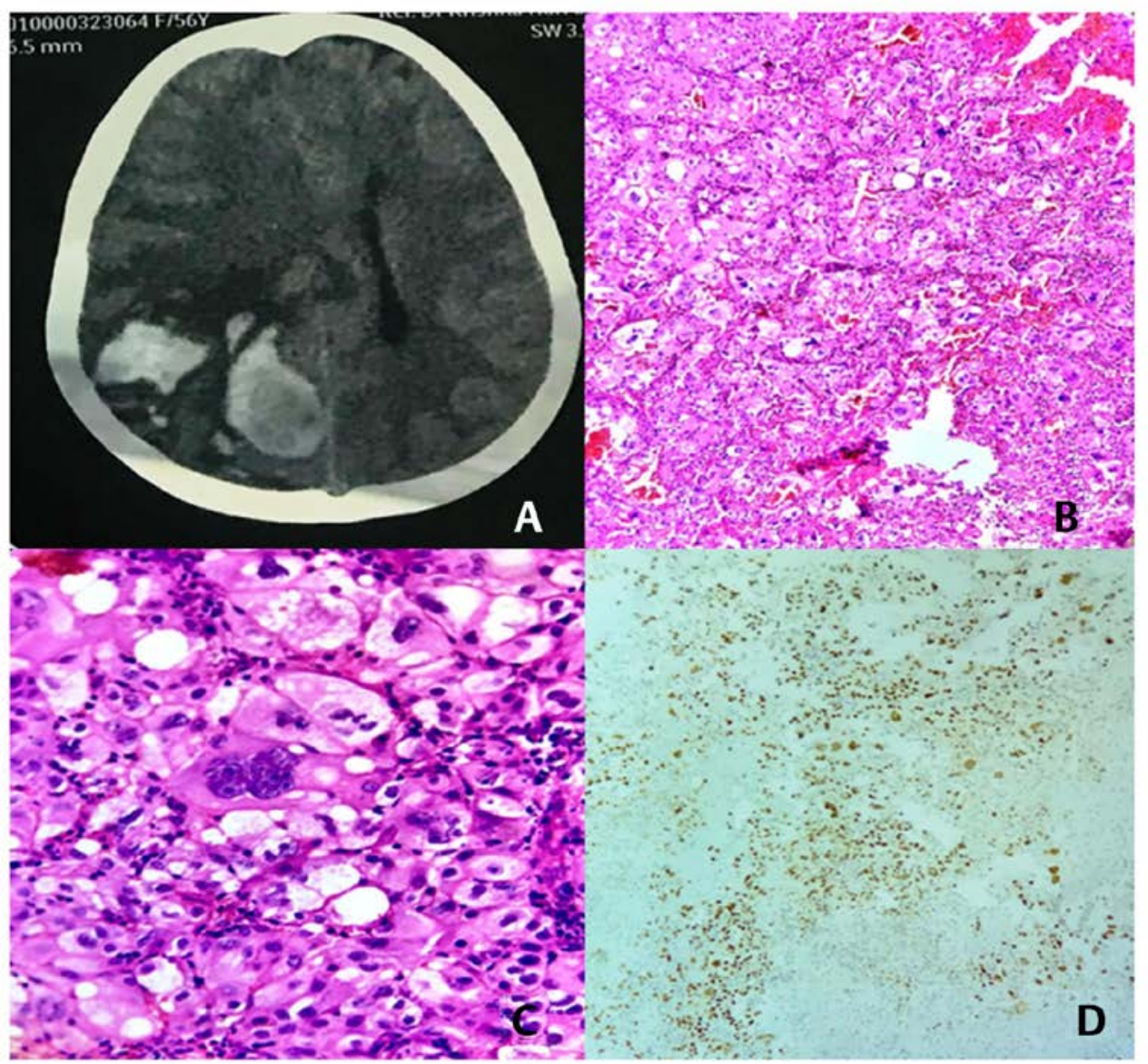

Fig. 1 (A) CT scan showing a heterogeneous hypodense lesion in right parietal lobe. (B, C) Microphotograph showing discohesive sheets of large pleomorphic cells having abundant eosinophilic cytoplasm, bi and multinucleate cells, and interstitial hemorrhage. (H \& E, x100, x400, respectively); (D) Tumors cells showing immunoreactivity for TTF-1 (x100).

sections showed gliosis and edematous brain parenchyma with intraparenchymal hemorrhage. In between the hemorrhage, there were discohesive sheets of large pleomorphic cells having abundant eosinophilic cytoplasm as well as bi and multinucleate cells present. Lymphocytic infiltrate was seen in between these cells. Small foci of necrosis and atypical mitosis were also noted, favoring neoplastic pathology ( - Fig. 1B, C). On performing IHC, tumor cells were positive for PanCK, CK7, TTF-1, Napsin A, EMA, and Vimentin. These tumor cells were negative for GFAP, S-100, Neu N, CD68, Synaptophysin, Chromogranin, CDX2, PAX-8, Beta-HCG, and GATA3. $\mathrm{K}_{\mathrm{i}} 67$ index was 20\% (-Fig. 1D). On the basis of morphology and IHC, case was diagnosed as metastatic carcinoma lung-pleomorphic giant cell variant. So, after histopathological diagnosis, CT of chest and abdomen was done, which revealed a rounded heterogeneously enhancing area of parenchymal consolidation, measuring $2.7 \times 2.7 \times 2 \mathrm{~cm}$, in left lower lobe of lung as well as a mildly prominent lymph node, measuring $12.5 \times 11 \mathrm{~mm}$, in the left hilum, suggesting a neoplastic etiology. In abdomen, there was a hypodense thrombus completely filling in and obliterating the lumen of left external iliac vein, extending superiorly into left common iliac vein and further into infrarenal inferior vena cava, indicating deep venous thrombosis. The patient was therefore finally diagnosed as PC of the lung with a single brain hemorrhagic metastasis and multiple thrombi. The patient was subsequently referred to the oncology department, where six cycles of chemotherapy (carboplatin day 1 and gemcitabine $800 \mathrm{mg} / \mathrm{m} 2$ on days 1 and 8 , repeated every 3 weeks). She showed a good clinical outcome with chemotherapy.

\section{Discussion}

Lung cancer can be classified into two types: small-cell lung carcinoma and nonsmall-cell lung carcinoma (NSCLC). Pulmonary PC is defined as a poorly differentiated NSCLC containing at least $10 \%$ sarcomatoid components of spindle or giant cells, or a carcinoma consisting solely of sarcomatoid components. ${ }^{3}$ The male to female ratio of pleomorphic carcinoma varies from 3:1 to $6: 1$, with the median age of presentation being 59 years. ${ }^{4}$ However, the patient in the current report was a 56-year-old female. Sixty percent of these tumors are present as a peripheral mass without endobronchial involvement, making it difficult, in some cases, to achieve a diagnosis with bronchoscopic examination or endobronchial biopsy. Approximately $50 \%$ of the cases 
present at stage III or IV, with chest wall invasion involving approximately $25 \%$ of the cases. ${ }^{5}$ Our case is unique in its presentation, having symptomatic brain metastasis prior to the discovery of the pulmonary lesion.

In cancer patients, causes of ICH are various, such as coagulation disorders, complications of anticancer treatment, hypertension, and cirrhosis. We should exclude these factors prior to defining tumor-related $\mathrm{ICH}^{6}{ }^{6}$ The appearance of primary brain tumors and of brain metastases is often quite similar, making it difficult to distinguish between them, especially on neuroimaging. Tumor-related ICH should be suspected if in neuro images, the ICH has surrounding edema, an enhanced solid mass within or near the $\mathrm{ICH}$, a high-or low-density neoplastic core, or small, multifocal clots usually at the margin of the tumor; this is similar to our case. ${ }^{1,7}$ Symptoms and presentation of brain metastasis as well as a high-grade primary brain tumors such as glioblastoma can also be quite similar, but patient prognosis can be quite different. Pathologists should be familiar with this unusual subtype of lung cancer and its behavior; also, physicians should be aware of the appearance of metastatic symptoms of sarcomatoid carcinoma, such as neurological symptoms, which can be prominent and misleading to other differential diagnoses because prompt diagnosis may facilitate earlier clinical intervention, leading to prolonged survival. There were various theories regarding etiology of intratumoral hemorrhage: endothelial proliferation with vascular obliteration, vessel compression and/or distortion due to rapid tumor growth, vessel necrosis, invasion of vessel walls by the tumor, and increased venous pressure associated with increased intracranial pressure. ${ }^{8}$ In the case reported by Hagihara et al, intracerebral multiple metastases were detected with hemorrhage, but the primary pulmonary giant cell cancer showed no hemorrhage. This same feature was also noted in our study, that is, no hemorrhage in primary pulmonary tumor in imaging, implying that the same tumor type does not produce the same result in different organs. The brain may have a stronger bleeding tendency than the lung. ${ }^{9}$ Koenig et al reported a case of recurrent hemorrhagic strokes associated with paraneoplastic disseminated intravascular coagulation (DIC) due to occult large cell carcinoma. In our patient, we also found evidence of DIC; we could not ignore this potential problem. ${ }^{10}$ Sometimes, paraneoplastic phenomena such as a hypercoagulable state with venous thrombosis and increased venous pressure, leading to the rupture of a venule, might play a role in the repeated ICHs of the metastatic tumors.

The National Comprehensive Cancer Network recommends neoadjuvant chemotherapy combined with surgical treatment or surgical treatment combined with adjuvant chemotherapy in NSCLC cases with brain metastasis (TNM category $\mathrm{T} 1-2, \mathrm{~N} 0-1, \mathrm{~T} 3 \mathrm{~N} 0$ if the metastasis can be controlled). In the current case, patient presents with an emergency, so no neoadjuvant chemotherapy was given, but after surgery, patient was put on adjuvant chemotherapy when an accurate diagnosis was made by biopsy.

\section{Conclusion}

Although pulmonary PC is an extremely uncommon aggressive neoplasm, it has the tendency to bleed in the brain. The characteristics of rapid growth and easy bleeding with early hematogenous spread may allow ICH to be discovered prior to the detection of lung cancer. A neuroradiologist and pathologist should be aware and familiar with metastatic presentation of this uncommon lung cancer. Further study of detailed factors affecting tumor bleeding requires more cases and animal model simulation.

\section{Funding \\ None.}

\section{Conflict of Interest}

None declared.

\section{References}

1 Schrader B, Barth H, Lang EW, et al. Spontaneous intracranial haematomas caused by neoplasms. Acta Neurochir (Wien) 2000;142(9):979-985

2 Shoji F, Maruyama R, Okamoto T, et al. Long-term survival after an aggressive surgical resection and chemotherapy for stage IV pulmonary giant cell carcinoma. World J Surg Oncol 2005;3:32

3 Travis WD, Brambilla E, Burke AP, et al. WHO Classification of Tumours of the Lung, Pleura, Thymus and Heart. 4th ed. Lyon: IARC Press; 2015

4 Rossi G, Cavazza A, Sturm N, et al. Pulmonary carcinomas with pleomorphic, sarcomatoid, or sarcomatous elements: a clinicopathologic and immunohistochemical study of 75 cases. Am J Surg Pathol 2003;27(3):311-324

5 Travis WD, Travis LB, Devesa SS. Lung cancer. Cancer 1995;75(1(Suppl):191-202

6 Wu DS, Wu CH, Hwang SN, et al. Repeated intracerebral hemorrhage as a presentation of pulmonary giant cell carcinoma with brain metastases. Formosan J Surg 2014;47:145-149

7 Little JR, Dial B, Bélanger G, Carpenter S. Brain hemorrhage from intracranial tumor. Stroke 1979;10(3):283-288

8 Kondziolka D, Bernstein M, Resch L, et al. Significance of hemorrhage into brain tumors: clinicopathological study. J Neurosurg 1987;67(6):852-857

9 Hagihara N, Abe T, Wakamiya T, Sugita Y, Watanabe M, Tabuchi K. A case of brain metastasis from pulmonary giant cell carcinoma. Kurume Med J 2010;57(1-2):39-41

10 Koenig MA, Maleszewski J, Winters B. Multiple hemorrhagic strokes from DIC associated with occult large cell carcinoma. Neurocrit Care 2006;5(3):210-212 